\title{
Conjugated linoleic acid feeding during gestation and suckling periods increases antibodies concentration in rat milk
}

\author{
C. Ramírez-Santana ${ }^{1,2}$, F. J. Pérez-Cano ${ }^{1}$, M. Castell ${ }^{1}$, M. C. López-Sabater ${ }^{2,3}$, \\ M. Rodríguez-Palmero ${ }^{4}$ C. Castellote ${ }^{1,2}$ and A. Franch ${ }^{1,2}$ \\ ${ }^{1}$ Department of Physiology, University of Barcelona, Barcelona, Spain, ${ }^{2}$ CIBER in Epidemiology and Public Health \\ (CIBERESP), Spain, ${ }^{3}$ Department of Nutrition and Food Science, Faculty of Pharmacy, University of Barcelona, Barcelona, \\ Spain and ${ }^{4}$ Research Department, Ordesa Group, Scientific Park of Barcelona, Barcelona, Spain
}

Conjugated linoleic acid (CLA) consists of a mixture of isomers of linoleic acid, of which cis $9, \operatorname{trans} 11(c 9, t 11)$ and trans 10, cis 12 $(t 10, c 12)$ predominate. These compounds induce beneficial effects on health and it has been suggested that they may modulate immune responses. Breast milk has Ig and many other bioactive molecules, such as growth factors, cytokines, nucleotides, cellular components and lipids, which promote maturation of the developing immune system. The aim of this study was to determine the effect of feeding $1 \%$ CLA on rat milk antibodies concentration and CLA isomers content in pup's plasma.

Pregnant Wistar rats were obtained on day 7 of gestation and were immediately fed 1\% CLA pellet chow or standard chow. After delivering, CLA-diet went on during suckling period in a group of CLA mothers (group A), whereas other previous CLA-mothers moved on to standard diet (group B). Litters from group B mothers were daily administered 1\% (w/w) CLA by oral gavage. Litters from mothers fed standard chow during gestation were daily administered 1\% CLA (group C) or only vehicle (group D). The 1\% CLA isomer mixture used was approximately $80 \% c 9, t 11$ and $20 \% t 10, c 12$ from the total CLA isomers in oil ${ }^{(1)}$. CLA oil was kindly supplied by Loders Croklaan, Lipid Nutrition, Wormerveer, The Netherlands. Milk was collected from dams on days 2, 5, 9, 14 and 21 post-partum. Total milk was used for CLA isomers quantification by gas chromatography, whereas Ig determination was performed in milk whey by ELISA. ANOVA and post hoc comparisons (Scheffe's test) were performed. Significant differences were accepted at $P<0.05$.

At the end of suckling period, milk from dams fed CLA diet had higher content of CLA and IgA and IgG concentration than rats fed standard diet (Table 1). At that day, plasma of pups from groups A, B and C showed 9, 12 and 6 times higher content of the $c 9, t 11$ CLA isomer than that of group D pups (Table 2). Moreover, similar results were observed in plasma from 14-day-old pups, as well as in milk collected on days 2, 5, 9 and 14 post-partum. In conclusion, supplementation during gestation and suckling with an 80:20 $c 9, t 11: t 10, c 12$ CLA mix, enhances the production of the main Ig isotypes in Wistar rats' milk, which are transferred to pups, as well as CLA.

Table 1

\begin{tabular}{lcrrr}
\hline Milk Ig $(\mu \mathrm{g} / \mathrm{ml})$ & \multicolumn{2}{c}{ CLA diet } & \multicolumn{2}{c}{ Standard diet } \\
\cline { 2 - 5 } on day 21 & Mean & SEM & Mean & SEM \\
\hline IgA & $58.62^{*}$ & 9.80 & 29.78 & 4.30 \\
IgG & $273.40^{*}$ & 19.27 & 49.89 & 21.56 \\
IgM & 5.84 & 1.40 & 5.07 & 0.61 \\
\hline$* P<0.05 v$ standard diet $n=4-7$ dams per group & &
\end{tabular}

Table 2

\begin{tabular}{lcccr}
\hline CLA isomers & \multicolumn{2}{c}{$c 9, t 11$} & & $t 10, c 12$ \\
\cline { 2 - 4 } Group & Mean & SEM & Mean & SEM \\
\hline A & 1.34 & 0.01 & 0.21 & 0.01 \\
B & 1.78 & 0.05 & 0.13 & 0.01 \\
C & 0.90 & 0.01 & 0.05 & 0.00 \\
D & 0.15 & 0.01 & & N.D. \\
\hline
\end{tabular}

N.D., non-detectable. Both CLA isomers content in pups' plasma are significantly different among all groups (one-way ANOVA, $P<0.001), n=10$.

1. Ramírez-Santana C, Castellote C, Castell M et al. (2009) J Nutr 139, 76-81. 